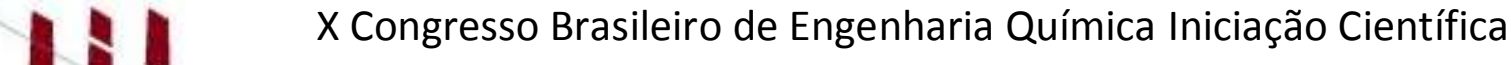 \\ "Influência da pesquisa em Engenharia Química no desenvolvimento tecnológico e industrial brasileiro" \\ Universidade Federal Rural do Rio de Janeiro Universidade Severino Sombra Vassouras - RJ-Brasil
}

\section{INFLUÊNCIA DA COMPOSIÇÃO DO MEIO DE CULTIVO NA PRODUÇÃO DE ÁCIDO CÍTRICO POR Yarrowia lipolytica}

\author{
DA SILVA* ${ }^{1}$, R. S.; DA SILVA ${ }^{2}$, L. V.; AMARAL ${ }^{3}$, P.F.F \\ ${ }^{1}$ Bolsista CNPq da UFRJ $\quad{ }^{2}$ Docente do DTA/UFRRJ $\quad{ }^{3}$ Docente do DEB/UFRJ \\ Departamento de Engenharia Bioquímica - Universidade Federal do Rio de Janeiro \\ Endereço - UFRJ, Av. Athos da Silveira Ramos, 149 - Bloco E, lab 103 - Ilha do Fundão - Rio de \\ Janeiro, CEP. 21941-909, RJ, \\ email: pamaral@eq.br
}

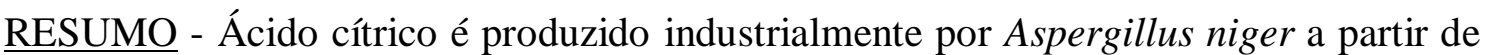
sacarose como fonte de carbono. A levedura Yarrowia lipolytica é capaz de produzir e excretar no meio de cultura este ácido, tendo glicerol como fonte de carbono. Diferentes níveis de minerais e vitaminas no meio de cultivo influenciam a produção do ácido pela levedura. Portanto, o objetivo deste trabalho foi avaliar a influência de meios minerais de composições diferentes e sua relação com a produção de ácido cítrico por Yarrowia lipolytica IMUFRJ 50682, a partir de glicerol P.A. como fonte de carbono. As condições experimentais foram: $250 \mathrm{rpm}$ de agitação a $28^{\circ} \mathrm{C}, 30 \mathrm{~g} / \mathrm{L}$ e $258,56 \mathrm{~g} / \mathrm{L}$ de glicerol, $0,1 \mathrm{~g} / \mathrm{L}$ de extrato de lêvedo para o primeiro experimento e a substituição deste por vitaminas específicas no segundo, conhecendo-se assim cada componente do meio. Utilizando extrato de lêvedo, a concentração máxima de ácido cítrico obtida foi de 8,97 $\mathrm{g} / \mathrm{L}$, enquanto no experimento constituído de vitaminas o maior valor foi de $1,93 \mathrm{~g} / \mathrm{L}$. $\mathrm{O}$ resultado mostrou que um meio composto pelo extrato levou a uma produção maior de ácido cítrico. Esses resultados demonstram que é possível obter uma maior concentração do ácido em questão a um menor custo de reagentes.
\end{abstract}

Palavras chave: glicerol, biodiesel, fermentação.

\section{INTRODUÇÃO}

Yarrowia lipolytica possui habilidade de produzir uma grande variedade de ácidos orgânicos utilizando várias fontes renováveis ou materiais residuais como matéria-prima. Como exemplo, podem ser citados os intermediários do ciclo do ácido tricarboxílico, tais como o ácido cítrico, o ácido isocítrico, o ácido alfa-cetoglutárico e o ácido pirúvico (BARTH E GAILLARDIN, 1997; FICKERS et al., 2005; KAMZOLOVA et al., 2003; RYMOWICZ et al., 2006).

A composição do meio de cultivo interfere no metabolismo de $Y$. lipolytica, que pode excretrar ácido cítrico sob condições de excesso de carbono e deficiência de nitrogênio no meio de cultivo (Finogenova et al., 2005; Rywińska et al., 2010; Silva, et al., 2010). Há também, simultaneamente, a produção de ácido isocítrico, em níveis que podem atingir valores acima de $50 \%$ do total de ácido produzido (Anastassiadis 
et al., 2002; Rymowicz et al., 2006; Venter et al., 2004). Esse ácido não é de interesse comercial, pois possui capacidade tamponante e quelante inferior ao ácido cítrico e dificulta a cristalização do ácido cítrico durante a etapa de purificação quando se apresenta em níveis acima de 5\% no meio de cultivo (Forster et al., 2007). A produção de ácido isocítrico por $Y$. lipolytica é influenciada pelo tipo de micro-organismo, fonte de carbono e concentração dos micronutrientes acessíveis à levedura (Venter et al. 2004; Rywińska et al. 2010).

Diferentes níveis de minerais e vitaminas no meio de cultivo também podem influenciar a produção de ácido cítrico pela levedura. As vitaminas intervêm em múltiplas reações enzimáticas de degradação ou síntese, ajudando a gerar energia indispensável para o trabalho celular. Atuando como coenzimas, são indispensáveis para transformações e renovações que condicionam a vida celular.

Portanto, o objetivo deste trabalho foi avaliar a influência de meios minerais de composições diferentes e sua relação com a produção de ácido cítrico por Yarrowia lipolytica IMUFRJ 50682, a partir de glicerol P.A. como fonte de carbono.

\section{MATERIAIS E MÉTODOS}

\section{Micro-organismo e condições de cultivo}

O micro-organismo utilizado no trabalho foi a cepa selvagem Yarrowia lipolytica IMUFRJ 50682 isolada de um estuário no Rio de Janeiro, Brasil (HAGLER e MENDONÇA-HAGLER, 1981) e identificada no Instituto de Microbiologia do Centro de Ciências da Saúde da Universidade Federal do Rio de Janeiro. As células foram conservadas por repiques regulares em tubos de ensaio com meio YPD, contendo (em p/v): extrato de lêvedo $1 \%$, peptona $2 \%$, glicose $2 \%$ e Agaragar $2 \%$ sob refrigeração $4{ }^{\circ} \mathrm{C}$.

\section{Inóculo e Amostragem}

A partir dos tubos contendo as células preservadas em meio sólido YPD inoculavase, de forma estéril, $200 \mathrm{~mL}$ de meio de cultivo YPD em frascos de erlenmeyers de 500 $\mathrm{mL}$. Após crescimento por 72 horas em incubador rotatório a $28{ }^{\circ} \mathrm{C}$ e $160 \mathrm{rpm}$, as células eram centrifugadas de forma estéril e resuspensas, servindo como inóculo dos experimentos que serão descritos nos itens posteriores. O volume centrifugado desse préinóculo era suficiente para se obter uma concentração inicial de $1 \mathrm{~g} / \mathrm{L}$ de células.

Durante a condução dos experimentos, foi realizada uma amostragem por dia durante 5 dias de cultivo: uma alíquota era convenientemente diluída para leitura da absorvância em espectrofotômetro para obtenção da concentração celular e a outra alíquota $(5 \mathrm{~mL})$ era centrifugada a $3000 \mathrm{rpm}$ durante 5 minutos, sendo o sobrenadante congelado $\left(-4^{\circ} \mathrm{C}\right)$ para posterior análise da produção de ácido cítrico e isocítrico, dosagem de glicerol e determinação do $\mathrm{pH}$, sendo ambas retiradas com o auxílio de pipetas estéreis e de forma asséptica em câmara de fluxo laminar.

\section{Condições de cultivo}

Para o primeiro experimento, o meio de cultivo foi preparado com $30 \mathrm{~g} / \mathrm{L}$ de glicerol P.A. e $0,1 \mathrm{~g} / \mathrm{L}$ de extrato de lêvedo em meio mineral tamponado, constituído de(em g/L): $\mathrm{KH}_{2} \mathrm{PO}_{4}: \quad 12 ; \quad \mathrm{Na}_{2} \mathrm{HPO} 4.7 \mathrm{H}_{2} \mathrm{O}: \quad 22,66$; $\mathrm{MgSO}_{4} .7 \mathrm{H}_{2} \mathrm{O}: 1,5 ; \mathrm{CaCl}_{2} .2 \mathrm{H}_{2} \mathrm{O}: 0,2 ; \mathrm{ZnSO}_{4} .7$ $\mathrm{H}_{2} \mathrm{O}: 0,02 ; \mathrm{FeCl}_{3}: 0,09 ; \mathrm{MnSO}_{4} \cdot \mathrm{H}_{2} \mathrm{O}: 6 \times 10^{-8}$. Todos os experimentos foram realizados em frascos de Erlenmeyer de $1000 \mathrm{~mL}$ contendo $400 \mathrm{~mL}$ de meio de cultivo, a $28^{\circ} \mathrm{C}$ e $250 \mathrm{rpm}$ de agitação em incubador rotatório por 5 dias com amostragem diária para crescimento celular, $\mathrm{pH}$ e dosagem de ácidos cítrico e isocítrico.

Para o segundo experimento, o meio de cultivo foi preparado com 258,56 g/L de glicerol P.A. e o seguinte meio mineral adicionado de vitaminas constituído de(em $\mathrm{g} / \mathrm{L}): \mathrm{KH}_{2} \mathrm{PO}_{4}-0,7 ; \mathrm{MgSO}_{4} \cdot 7 \mathrm{H}_{2} \mathrm{O}$ - 0,35; $\mathrm{CaCl}_{2} \quad-\quad 0,1 ; \quad \mathrm{FeSO}_{4} \cdot 7 \mathrm{H}_{2} \mathrm{O} \quad-\quad 0,139$; $\mathrm{ZnSO}_{4} .7 \mathrm{H}_{2} \mathrm{O}-0,021 ; \mathrm{MnSO}_{4} \cdot 4 \mathrm{H}_{2} \mathrm{O}-0,11$; $\mathrm{FeSO}_{4} \cdot 7 \mathrm{H}_{2} \mathrm{O}-0,139 ; \mathrm{NH}_{4} \mathrm{Cl}-3 ; \mathrm{CaCO}_{3}-3$; $\mathrm{CuSO}_{4} .5 \mathrm{H}_{2} \mathrm{O}-0,001 ; \mathrm{H}_{3} \mathrm{BO}_{3}-0,04 \mathrm{~g} / \mathrm{L}$; $\mathrm{CoSO}_{4} \cdot 7 \mathrm{H}_{2} \mathrm{O}-4 \mathrm{mg} / \mathrm{L} ; \mathrm{NaCl}-0,1 \mathrm{~g} / \mathrm{L}$; $\mathrm{Na}_{2} \mathrm{MoO}_{4} \cdot 2 \mathrm{H}_{2} \mathrm{O}-0,2 \mathrm{mg} / \mathrm{L} ; \mathrm{KI}-0,1 \mathrm{~g} / \mathrm{L}$; 
Tiamina-HCl - 2 mg/L; Biotina - 0,25 mg/L; Piridoxina- $\mathrm{HCl}$ - 0,625 mg/L; Pantotenato de cálcio - 0,625 mg/L; Ácido nicotínico - 0,5 $\mathrm{mg} / \mathrm{L}$.

\section{Métodos Analíticos}

Quantificação do crescimento celular: O crescimento celular foi determinado em espectrofotômetro por medidas de densidade óptica a $570 \mathrm{~nm}$ e esses valores foram convertidos para $\mathrm{mg}$ p.s./mL usando-se um fator de conversão previamente determinado pelas curvas de peso seco.

Quantificação de ácido cítrico e isocítrico: Foram realizadas por cromatografia líquida de alta eficiência (CLAE-HPLC), com sistema de dupla bomba, e um detector ultravioleta com lâmpada de zinco a $214 \mathrm{~nm}$. A coluna analítica utilizada foi a de polímero de octadecilsilano (YMC-Pack ODSAQ, S-5 $\mu \mathrm{m}, 12 \mathrm{~nm})$. A amostra foi eluída pela coluna a uma vazão de $0,5 \mathrm{~mL} / \mathrm{min}(0,45 \mathrm{~mL} / \mathrm{min}$ de tampão $\mathrm{KH}_{2} \mathrm{PO}_{4}$ e $0,05 \mathrm{~mL} / \mathrm{min}$ de metanol 70 $\%)$. A temperatura da coluna foi mantida constante e igual a $35^{\circ} \mathrm{C}$ em forno.

Determinação do $\mathrm{pH}: \mathrm{O}$ pH do meio de cultivo livre de células foi determinado utilizando-se um pHmetro digital previamente calibrado com tampões padrão 7,0 e 4,0 na temperatura ambiente $\left(25^{\circ} \mathrm{C}\right)$.

\section{Métodos Analíticos}

Para comparação entre todos os ensaios, parâmetros de produção, como rendimento de ácido cítrico a partir do consumo de glicerol (YP/S), fator de conversão de glicerol em células (YX/S), rendimento de ácido cítrico em relação à biomassa formada (YP/X), produtividade volumétrica de ácido cítrico (QAC) e razão entre a produção de ácido cítrico e de isocítrico (RAC/AIC) foram determinados pelas seguintes equações:

$$
\begin{aligned}
& Y_{P / S}=\frac{P_{A C}}{S f-S o} \\
& Y_{P / X}=\frac{P_{A C}}{X f-X o} \\
& Y_{X / S}=\frac{X f-X o}{S f-S o} \\
& Q_{A C}=\frac{P_{A C}}{V^{*} t} \\
& R_{A C / A I C}=\frac{P_{A C}}{P_{A I C}}
\end{aligned}
$$

Onde, $\mathrm{P}_{\mathrm{AC}}$ é a concentração de ácido cítrico máxima produzida; $\mathrm{P}_{\mathrm{AIC}}$ é a concentração de ácido isocítrico no tempo $t$ de máxima produção de ácido cítrico; $\mathrm{S}_{\mathrm{f}}$ é a concentração de glicerol no tempo $t$ de máxima produção de ácido cítrico; $\mathrm{S}_{\mathrm{o}}$ é a concentração inicial de glicerol; $X_{f}$ é a concentração celular no tempo $t$ de máxima produção de ácido cítrico; $\mathrm{X}_{\mathrm{o}}$ é a concentração celular inicial; $\mathrm{V}$ o volume inicial da cultura líquida e $t$ é o tempo de fermentação até a máxima concentração de ácido cítrico obtida.

\section{RESULTADOS}

A partir de dois meios de cultivo, um formulado com o extrato de levedura e o outro com vitaminas específicas, tem-se a seguinte tabela para produção de ácidos:

\section{Tabela 1 - Produção de ácidos nos dois meios analisados}

\begin{tabular}{|c|c|c|c|c|}
\hline $\begin{array}{c}\text { Condição } \\
\text { experimental }\end{array}$ & $\begin{array}{c}\mathbf{P}_{\mathrm{AC}} \\
(\mathrm{g} / \mathrm{L})\end{array}$ & $\begin{array}{c}\mathbf{P}_{\text {AIC }} \\
(\mathrm{g} / \mathrm{L})\end{array}$ & $\mathbf{R}_{\text {AC/AIC }}$ & $\begin{array}{c}\mathbf{P}_{\text {CA+P }} \\
(\mathrm{g} / \mathrm{L})\end{array}$ \\
\hline $\begin{array}{c}\text { Extrato de } \\
\text { lêvedo }\end{array}$ & $\mathbf{8 , 9 7}$ & $\mathbf{7 , 6 8}$ & $\mathbf{1 , 1 7}$ & $\mathbf{1 6 , 6 5}$ \\
\hline $\begin{array}{c}\text { Meio com } \\
\text { vitaminas }\end{array}$ & $\mathbf{1 , 9 7}$ & $\mathbf{9 , 6 7}$ & $\mathbf{0 , 2 0}$ & $\mathbf{1 1 , 6 4}$ \\
\hline
\end{tabular}


A partir da Tabela 1, pode-se observar que a produtividade e a concentração máxima obtida de ácido cítrico foram maiores para o ensaio com o extrato. Além da maior produção de ácido cítrico, a produção de ácido isocítrico foi próxima entre ambos, explicando o motivo da razão de produção de ácidos deste ensaio ser melhor.

A Tabela 2 consiste da análise dos fatores de conversão, variação de $\mathrm{pH}$ e produtividade dos dois meios utilizados.

Tabela 2 - Fatores de conversão, variação de $\mathrm{pH}$ e produtividade

\begin{tabular}{|c|c|c|c|c|c|c|}
\hline $\begin{array}{c}\text { Condição } \\
\text { exp. }\end{array}$ & $\begin{array}{c}\mathbf{Y}_{\mathrm{P} / \mathrm{S}} \\
(\mathrm{g} / \mathrm{g})\end{array}$ & $\begin{array}{c}\mathrm{Y}_{\mathrm{X} / \mathrm{s}} \\
(\mathrm{g} / \mathrm{g})\end{array}$ & $\begin{array}{c}\mathrm{Y}_{\mathrm{P} / \mathrm{X}} \\
(\mathrm{g} / \mathrm{g})\end{array}$ & $\mathbf{p H}_{\mathbf{0}}$ & $\mathbf{p H}_{\mathrm{f}}$ & $\begin{array}{c}\mathrm{Q}_{\mathrm{CA}} \\
(\mathrm{g} / \mathrm{L} \cdot \mathrm{h})\end{array}$ \\
\hline $\begin{array}{c}\text { Extrato } \\
\text { de lêvedo }\end{array}$ & $\mathbf{0 , 3 9}$ & $\mathbf{0 , 1 5}$ & $\mathbf{2 , 6 5}$ & $\mathbf{6 , 7}$ & $\mathbf{3 , 7}$ & $\mathbf{0 , 0 9}$ \\
\hline $\begin{array}{c}\text { Meio com } \\
\text { vitaminas }\end{array}$ & $\mathbf{0 , 0 8}$ & $\mathbf{0 , 2 6}$ & $\mathbf{3 , 3 5}$ & $\mathbf{5 , 7}$ & $\mathbf{5 , 0}$ & $\mathbf{0 , 0 2}$ \\
\hline
\end{tabular}

Pela Tabela 2, observa-se uma variação de $\mathrm{pH}$ diferente nos dois ensaios, denotando uma influência do meio de cultivo na produção do ácido cítrico. A produtividade do ensaio utilizando $\mathrm{o}$ extrato foi aproximadamente quatro vezes maior que no outro ensaio, também demonstrando um meio de cultivo mais otimizado para a produção do ácido de interesse.

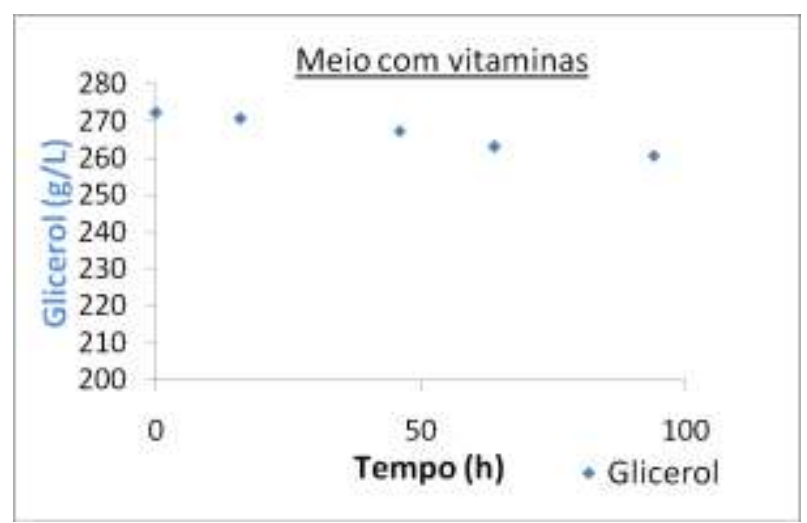

Figura 1 - Consumo de glicerol do ensaio com vitaminas

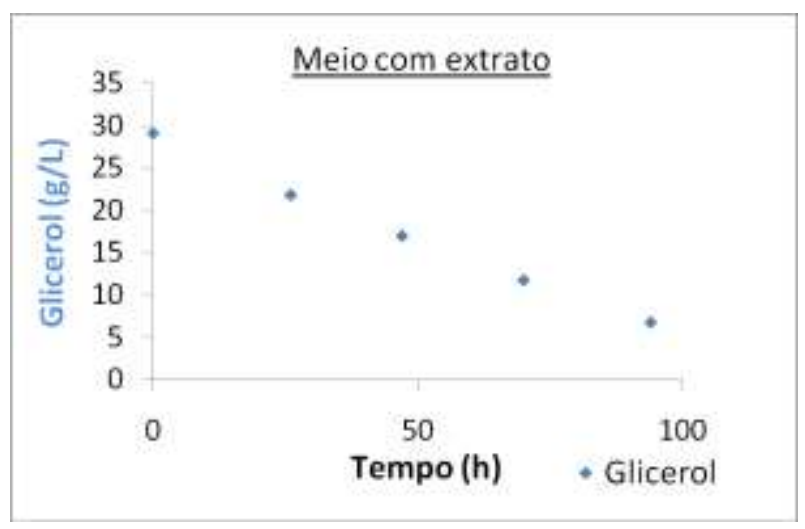

Figura 2 - Consumo de glicerol do ensaio com extrato de lêvedo

Comparando os dados de consumo de glicerol nos dois ensaios, há maior consumo da substância no ensaio a partir de extrato de levedura, tendo o ensaio com a vitaminas consumido aproximadamente a metade do valor do outro ensaio, apesar da concentração mutio maior de glicerol neste ensaio. Dessa forma, pode-se economizar na produção de meios utilizando o que demanda menor uso do glicerol total no meio.

\section{CONCLUSÕES}

Através deste estudo, é possível perceber que o meio composto pelo extrato de levedura como fonte de vitaminas e nitrogênio gerou uma maior produção de ácido cítrico por Yarrowia lipolytica IMUFRJ 50682. Esses resultados demonstram que é possível obter uma maior concentração do ácido em questão a um menor custo de reagentes. $O$ ácido isocítrico é um composto indesejado no processo de obtenção de ácido cítrico por processo fermentativo, uma vez que dificulta sua recuperação na etapa de purificação, porém é de interesse o estudo em formas de se converter este ácido em ácido cítrico para aumentar a produção final de citrato.

\section{REFERÊNCIAS}

ANASTASSIADIS, S.; AIVASIDIS, S.; WANDREY, C. Citric acid production by Candida strains under intracellular nitrogen limitation. Appl Microbiol Biotechnol, v. 60, p. 81-87, 2002. 
BARTH, G. \& GAILLARDIN, C. Physiology and genetics of the dimorphic fungus Yarrowia lipolytica. FEMS Microbiology Reviews, v. 19, p. 21-237, 1997.

FINOGENOVA, T. V.; MORGUNOV, I. G.; KAMZOLOVA, $\quad$ S. $\quad$ V; CHERNYAVSKAYA, O. G. Organic Acid Production by the Yeast Yarrowia lipolytica: A Review of Prospects. Applied Biochemistry and Microbiology, v. 41(5), p. 418-425, 2005.

FÖRSTER, A.; AURICH, A.; MAUERSBERGER, S.; BARTH, G. Citric acid production from sucrose using a recombinant strain of the yeast Yarrowia lipolytica. Applied Microbial and Cell Physiology. Appl Microbiol Biotechnol DOI 10.1007/s00253-007-0958-0, 2007.

HAEGLER, A.N.\& MENDONÇA-HAEGLER, L.C. Yeasts from marine and estuarine waters with different levels of pollution in the state of Rio de Janeiro, Brazil. Appl. Environ. Microbiol., v. 41, n. 1, p. 173178, 1981.

RYMOWICZ, W.; RYWINSKA, A.; AROWSKA, B.; JUSZCZYK, P. Citric Acid Production from Raw Glycerol by Acetate Mutants of Yarrowia lipolytica. Chem. Pap., v. 60 (5), p. 391-394, 2006.

RYWIŃSKA, A.; RYMOWICZ, W.; MARCINKIEWICZ, M. Valorization of raw glycerol for citric acid production by Yarrowia lipolytica yeast. Electronic Journal of Biotechnology, v. 13, n. 4, 2010.

SILVA, L.V. ; RIBEIRO, R. ; AMARAL, P. F. F. ; COELHO, M. A. Z. ; PESSOA, F. L. P. Potential application of raw glycerol from biodiesel in citric acid production by Yarrowia lipolytica. Chemical Engineering Transactions, v. 20. p. 211216, 2010.

VENTER, T.; KOCK, J. L. F.; BOTES, P. J.; SMIT, M. S.; HUGO, A. E JOSEPH, M. Acetate Enhances Citric Acid Production by Yarrowia lipolytica When Grown on Sunflower Oil. Systematic and Appied Microbiology, v. 27, p. 135-138, 2004. 